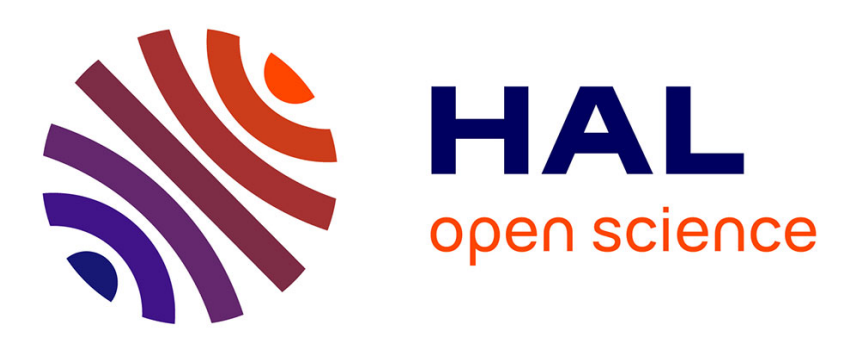

\title{
Oleylamine-assisted solvothermal synthesis of copper antimony sulfide nanocrystals: Morphology and phase control
}

\author{
M Bella, C. Rivero, S. Blayac, H Basti, M C Record, Pascal Boulet
}

\section{- To cite this version:}

M Bella, C. Rivero, S. Blayac, H Basti, M C Record, et al.. Oleylamine-assisted solvothermal synthesis of copper antimony sulfide nanocrystals: Morphology and phase control. Materials Research Bulletin, 2017, 90, pp.188-194. 10.1016/j.materresbull.2017.02.036 . hal-01483318

\section{HAL Id: hal-01483318 \\ https://hal-amu.archives-ouvertes.fr/hal-01483318}

Submitted on 26 Apr 2018

HAL is a multi-disciplinary open access archive for the deposit and dissemination of scientific research documents, whether they are published or not. The documents may come from teaching and research institutions in France or abroad, or from public or private research centers.
L'archive ouverte pluridisciplinaire HAL, est destinée au dépôt et à la diffusion de documents scientifiques de niveau recherche, publiés ou non, émanant des établissements d'enseignement et de recherche français ou étrangers, des laboratoires publics ou privés. 


\title{
Oleylamine-assisted solvothermal synthesis of copper antimony sulfide nanocrystals: morphology and phase control
}

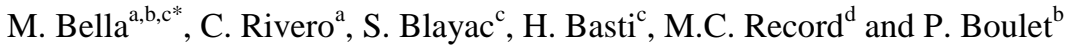 \\ a. STMicroelectronics, Rousset, France \\ b. Aix Marseille Université, CNRS, MADIREL UMR 7246, Marseille, France \\ c. Centre Microélectronique de Provence, Mines-Saint Etienne, Gardanne, France \\ d. Aix Marseille Université, CNRS, IM2NP UMR 7334, Marseille, France
}

We report a one-pot synthesis of copper antimony sulfide nanocrystals by a facile and easily scalable route using a surfactant assisted solvothermal method. Phase-pure and quasi-monodisperse $\mathrm{Cu}_{3} \mathrm{SbS}_{4}$ nanocrystals have successfully been synthetized by adjusting the sulfur to copper molar ratio as well as the surfactant to solvent volumetric ratio. Nanocrystals morphology and chemical composition are characterized. As synthetized and purified nanocrystals are formulated into a colloidal suspension in order to fabricate copper antimony sulfide films. Morphological, electrical and optical characterizations of famatinite films are conducted. The electrical resistivity of the as deposited films is comprised between $10 \Omega . \mathrm{cm}$ and $48 \Omega . \mathrm{cm}$. The optical gap is found to be indirect with a value of $0.82 \mathrm{eV}$.

\section{Introduction}

The successful synthesis of colloidal semiconducting nanocrystals (NCs) ink is a prerequisite step toward large-scale fabrication of conformable active devices for energy applications such as thermoelectrics and photovoltaics. In effect, colloidal nanocrystals are most suitable for large-scale deposition techniques such as inkjet and screen printing, provided that a large volume of phase pure nanomaterials are obtained. The prerequisites for printing conductive or semiconductive nanomaterials are, essentially size control, stability of the colloidal dispersion, and high nanoparticle loading (20 to $80 \mathrm{wt} \%)$. Consequently large scale and reproducible nanoparticles production has to be ensured when choosing the synthesis technique.

Copper antimony sulfide (CAS) materials have attracted a lot of attention lately due to their promising thermoelectric and photovoltaic properties ${ }^{1-5}$. In addition, since CAS are constituted of cheap, available and abundant elements they are envisaged as sustainable materials for energy conversion applications. In spite of these remarkable properties, there are only a few investigations that have been driven on the synthesis of CAS NCs, in particular on the famatinite compound $\left(\mathrm{Cu}_{3} \mathrm{SbS}_{4}\right)^{6-10}$. The elaboration method used in ${ }^{6-9}$ is the hot-injection one, which enables the synthesis of small batches of monodisperse nanocrystals of a few nanometers with precise phase control under controlled inert atmosphere. However, this method is hardly transferable for industrial, large-scale synthesis of phase pure monodisperse nanocrystals as it is necessary to precisely control the injection temperature as well as provide efficient mixing of the precursors in the hot solvent to yield homogeneous nucleation. An et al. have obtained large famatinite crystals and tetrahedrite NCs using a solvothermal approach $^{10}$, which is an efficient route towards the large-scale production of nanomaterials. However, the particles morphology and size were not controlled, which is not suitable for the fabrication of inks. Homogeneous dispersions with functionalized nanocrystals are preferable as they can be more easily dispersed in the solvent. In this paper, we thus propose a new method for the synthesis of phase pure and monodisperse famatinite nanocrystals by a surfactant assisted solvothermal route. This method enables the synthesis of functionalized spherical CAS nanocrystals by a versatile solvothermal method which is a step towards facile synthesis of large-scale phase pure CAS NCs. 
In this paper, the synthesis procedure for the obtention of phase pure, nearly monodisperse famatinite nanocrystals is described. Those nanocrystals morphology and chemical composition are then characterized via SEM, TEM techniques and EDX, XPS ones, respectively. Details on ink preparation and films fabrication methods are then given along with structural, optical and electrical characterization of CAS films.

\section{Experimental section}

Materials. Chemicals used for CAS nanoparticle synthesis were ethanol, distilled water, copper (II) chloride dihydrate $\left(\mathrm{CuCl}_{2} \cdot 2 \mathrm{H}_{2} \mathrm{O}>99.99 \%\right)$ from Acros Organics, elemental sulfur $(99.998 \%)$ and oleylamine (OLA technical grade, $70 \%$ ) from Sigma-Aldrich, antimony (III) chloride $(\mathrm{SbCl} 3 \geq 99.95 \%)$ from Alfa Aesar, which was stored in a glovebox under $\mathrm{N}_{2}$ atmosphere to avoid moisture. N-heptane (99\%), used for the preparation of the nanoparticle dispersion, was purchased from Sigma-Aldrich. All reagents were used as is without any further purification.

Synthesis and purification of $\mathbf{C u}_{3} \mathrm{SbS}_{4}$ NCs. The synthesis of $\mathrm{Cu}_{3} \mathrm{SbS}_{4}$ relied on the preparation of two solutions, one containing copper (II) chloride and antimony (III) chloride dissolved in absolute alcohol with a $\mathrm{Cu}: \mathrm{Sb}$ molar ratio of $1: 1$, and the other containing elemental sulfur dissolved in oleylamine which was thoroughly sonicated for $1 \mathrm{~h}$ to obtain a clear solution. Both solutions were then mixed and stirred for a few minutes. The mixture was then loaded into a $50 \mathrm{~mL}$ Teflon-liner and carefully sealed to prevent leakage and impurities formation from the reaction with oxygen during the synthesis. The final solution volume represented $80 \%$ of the reactor volume. The Teflon-liner was then inserted in a stainless steel autoclave, which was subsequently sealed and heated up to $150{ }^{\circ} \mathrm{C}$ and maintained at this temperature for $15 \mathrm{~h}$. The autoclave was then left to cool down to room temperature by natural convection.

The synthetized nanoparticles were then isolated and washed with ethanol and centrifuged (Heraeus centrifuge from Thermo Scientific) at $4000 \mathrm{rpm}$ for 5 minutes to remove the unreacted salts, reaction by-products and excess capping agent. The process was repeated until a clear supernatant was obtained. This step was followed by a final centrifugation after dispersion in $25 \mathrm{~mL}$ of $\mathrm{n}$-heptane and the addition of $25 \mathrm{~mL}$ of ethanol, necessary to precipitate the nanoparticles. The powder was then finally isolated and dried in oven under vacuum at $50{ }^{\circ} \mathrm{C}$ for $2 \mathrm{~h}$.

Different volumetric ratios of oleylamine/ethanol (1:1, 1:2 and 1:3) and different sulfur to copper molar ratios (1:0.4 and 1:0.75) were investigated.

Ink preparation, film deposition. The $50 \mathrm{wt} \%$ nanoparticle solution was obtained by dispersing the nanoparticles in n-heptane. Films were deposited by spin-coating, at $2000 \mathrm{rpm}$ for $20 \mathrm{~s}$, on a quartz substrate cleaned beforehand in an ultra-sonic bath with acetone and isopropanol consecutively. The process was repeated several times to obtain micrometer thick layers.

\section{Characterization}

Nanocrystals. X-Ray diffraction (XRD) analyses were done on the isolated and purified powders using a Siemens D5000 (Bruker) diffractometer $(\mathrm{Cu} K \alpha$ radiation at $0.15418 \mathrm{~nm})$. Scanning electron microscopy (SEM) images were recorded using a field-emission gun scanning electron microscope Ultra 55 from Zeiss. Energy-dispersive X-ray (EDX) analyses were performed using an EDAX X-ray detector from Oxford Instrument mounted on the scanning electron microscope. High resolution transmission electron microscopy (HRTEM) images were recorded with a Philips CM200 transmission electron microscope. The sample for TEM analyses was prepared by drop deposition of a $1 \mathrm{wt} \%$ suspension of CAS nanocrystals in n-heptane on a carbon-coated gold grid which was subsequently dried in air under atmospheric pressure and room temperature. X-ray photoelectron spectroscopy (XPS) analyses were conducted using an Axis Nova equipment.

Films. The electrical sheet resistance was measured using a 4-point probe equipment (Jandel). UV/VIS/NIR measurements were carried out with a spectrometer (UV 2600) from SHIMADZU. 


\section{Results and discussion}

Solvothermal synthesis of CAS nanocrystals. In the synthesis procedure reported by An et al. ${ }^{10}$, copper (I) chloride and thiourea are used as copper and sulfur sources, respectively. In our procedure, described in the experimental section, copper (II) chloride is preferred as it can easily be dissolved in ethanol. In order to obtain functionalized nanocrystals with a controlled morphology, a hydrophobic ligand molecule is added during the synthesis. Oleylamine is chosen for its ability to act both as a surfactant as well as a reducing agent. Sulfur is used instead of thiourea as it can easily be dissolved in oleylamine and is less harmful. Two solutions are prepared, one containing metal salts dissolved completely in ethanol and the other is an homogeneous sulfur-oleylamine solution. Upon mixing the two initial solutions, a dark brown precipitate is immediately observed. SEM pictures and elemental mapping through energy dispersive X-ray spectroscopy (EDS) indicate that this precipitate is mainly composed of large sulfur particles, a small amount of $\mathrm{Cu}-\mathrm{Sb}-\mathrm{S}$ particles being also present (Fig. 1).

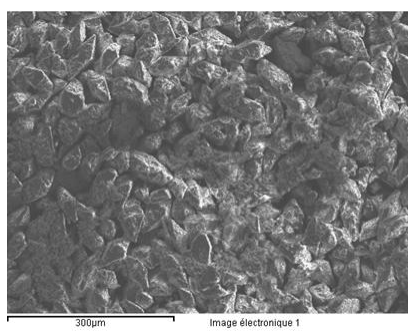

(a)

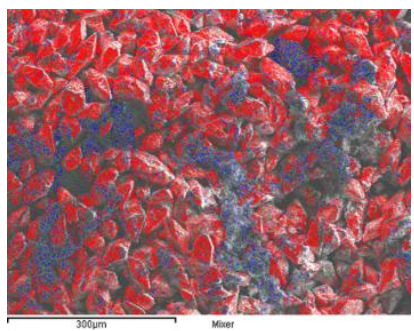

(b)

Fig. 1. Analysis of the precipitate formed upon mixing the initial solutions (metal salts in ethanol and sulfur in oleylamine) (a) SEM picture and (b) EDS mapping (sulfur in red, antimony in blue and copper in green)

The presence of large sulfur particles can be explained by the poor solubility of sulfur in ethanol. Nanocrystals (NCs) nucleation and growth is achieved by heating the heterogeneous mixture for 15 hours at $150{ }^{\circ} \mathrm{C}$. Heating time has a direct influence on the NCs synthesis yield due to the slow reaction kinetic using sulfur in oleylamine ${ }^{11-12}$. Each batch produces around two grams of functionalized nanocrystals. Based on the amount of reacted copper, an estimated yield around $90 \%$ is achieved with a heating time of $15 \mathrm{~h}$.

NCs characterization. The influence of surfactant to solvent ratio on the product morphology is investigated. Two ratios have been selected for this study, namely 1:1 (batch-1.1) and 1:3 (batch-1.3) volumetric ratios of oleylamine/ethanol. Nanocrystals from batch-1.1 are found to be quasi-monodisperse (Fig. 2.-a) with an average size of $20 \mathrm{~nm}$. Particles from batch-1.3 size dispersion is found to be highly polydisperse (Fig. 2.-b).

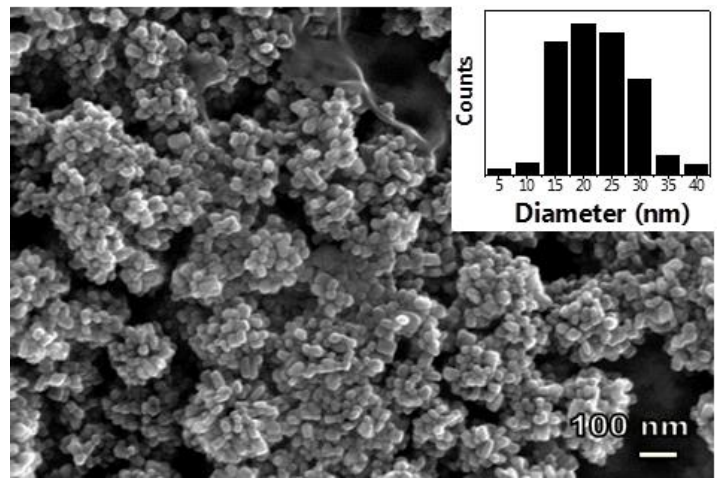

(a)

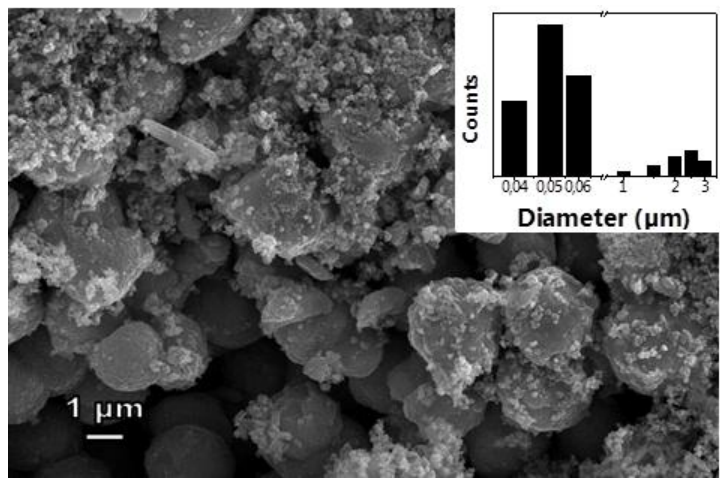

(b)

Fig. 2. SEM images of the synthetized nanoparticles with different surfactant/solvent volumetric ratios: (a) 1:1 and (b) 1:3 
Increasing the proportion of oleylamine induces better control of nanoparticle size. This can be attributed to the fact that oleylamine serves as both a coordinating solvent and a surfactant. The $-\mathrm{NH}_{2}$ group in oleylamine interacts with metal precursors and partakes in the nanoparticles formation. The ligand molecules bound to the nanoparticle surface not only control the growth of the particles during synthesis, but also prevent the aggregation of the nanoparticles. Specifically, lone pairs of electrons on the nitrogen atoms in the polar groups of oleylamine are donated to an sp hybrid orbital of the $\mathrm{Cu}_{2 \mathrm{p}}$ ions to form a $\mathrm{Cu}-\mathrm{N}$ coordination allowing the ligand molecule to control the growth rate of the nanoparticle through the limitation of the diffusion rate of metal precursors at the surface of the nanoparticle. Moreover, during nanoparticles growth stage, the surfactant reduces the surface tension $^{13}$ and avoids agglomeration through repulsive force between particles due to steric exclusion. Besides, as mechanical stirring is not possible in the autoclave, effective mass transport is ensured by the solvent reflux as it is heated in a closed vessel and above its boiling point. The product from batch-1.1 was chosen for further characterization as it is highly homogeneous and the synthesis procedure yields reproducible results. Figure 3 shows SEM (Fig. 3.-a) and TEM (Fig. 3.-b) micrographs for batch-1.1 nanoparticles produced with a molar ratio $\mathrm{S}: \mathrm{Cu}$ of 1:0.4. Nanoparticles average size is $20 \mathrm{~nm}$. HR-TEM picture (Fig. 3.-b) shows a highly crystalline isolated nanocrystal.

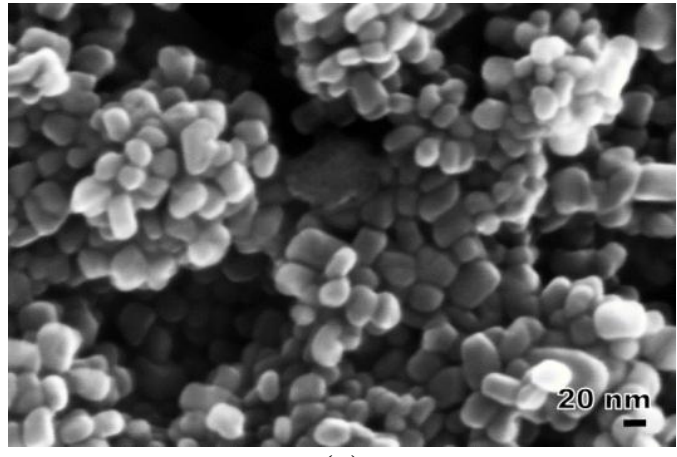

(a)

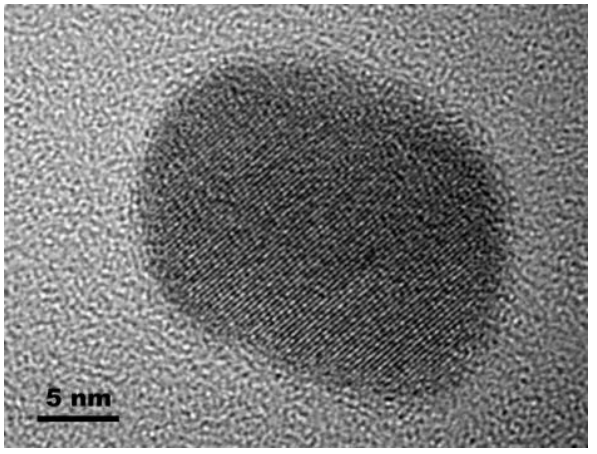

(b)

Fig. 3. SEM (a) and TEM images (b) of as synthetized famatinite nanocrystals

The crystallographic structure of the synthetized powder was investigated by means of X-ray diffraction. All the diffraction peaks in the XRD pattern (Fig. 4.) can be attributed to the tetragonal structure of $\mathrm{Cu}_{3} \mathrm{SbS}_{4}$ (JCPDS \# 01-071-0555). The recorded diffractogram is in good agreement with that calculated from the crystallographic data of $\mathrm{Cu}_{3} \mathrm{SbS}_{4}$ (space group $I \overline{4} 2 \mathrm{~m}$, with a $=5.38500 \AA$ and $\mathrm{c}=10,75400 \AA$ ) and represented as vertical bars in Fig. 4. No diffraction peaks corresponding to other ternary or binary phases in the $\mathrm{Cu}-\mathrm{Sb}-\mathrm{S}$ system can be detected in the XRD pattern. This observation confirms that no other crystalline phase than famatinite $\left(\mathrm{Cu}_{3} \mathrm{SbS}_{4}\right)$ is obtained with the synthesis route employed in this work.

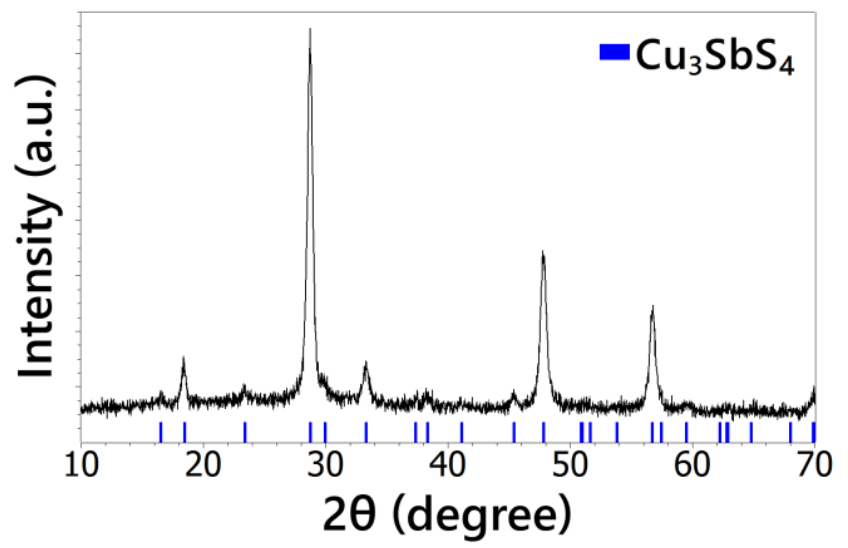

Fig. 4. XRD patterns of as prepared CAS nanocrystals synthetized with excess sulfur 
When the sulfur to copper molar ratio equals 1:0.75, which corresponds to the $\mathrm{Cu}_{3} \mathrm{SbS}_{4}$ stoichiometry, the synthesized product is a mix of famatinite $\left(\mathrm{Cu}_{3} \mathrm{SbS}_{4}\right)$ and tetraedrite $\left(\mathrm{Cu}_{12} \mathrm{Sb}_{4} \mathrm{~S}_{13}\right)$ phases. The XRD pattern of this product is represented in Fig. 5. The XRD pattern calculated from the crystallographic data of $\mathrm{Cu}_{12} \mathrm{Sb}_{4} \mathrm{~S}_{13}$ (space group $I \overline{4} 3 m, a=10.327 \AA$ ) is represented as red vertical bars whereas the calculated $\mathrm{XRD}$ pattern of $\mathrm{Cu}_{3} \mathrm{SbS}_{4}$ is represented in blue (Fig. 4). A Rietveld refinement has been performed to estimate the weight fraction of each crystalline component, which gives approximately $67 \%$ of famatinite and $33 \%$ of tetrahedrite. This study shows that, to obtain pure famatinite nanocrystals, sulfur excess is necessary in the initial mixing.

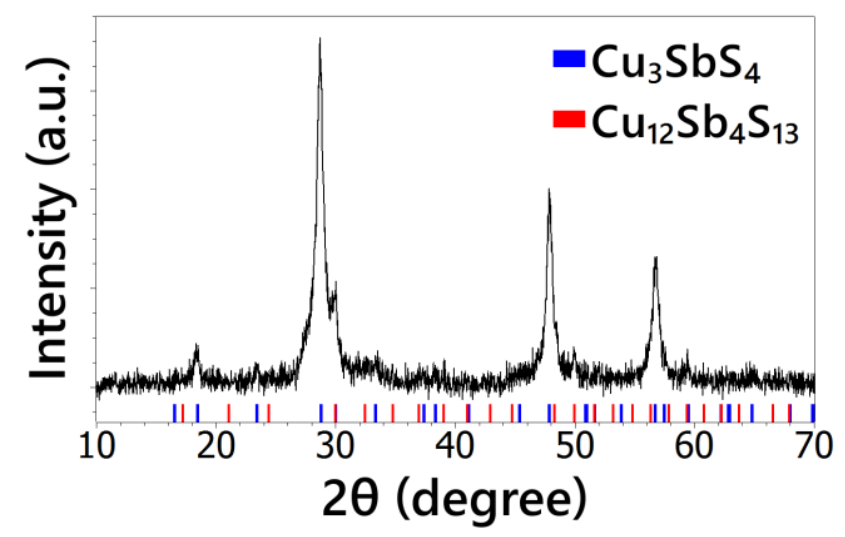

Fig. 5. XRD pattern of as prepared CAS nanocrystals synthetized with a sulfur to copper molar ratio of $1: 0.75$

The chemical structure of famatinite nanocrystals batch-1.1 is analyzed via X-ray photoelectron spectroscopy (XPS). The powder is found to be chlorine free (Fig. 6.).

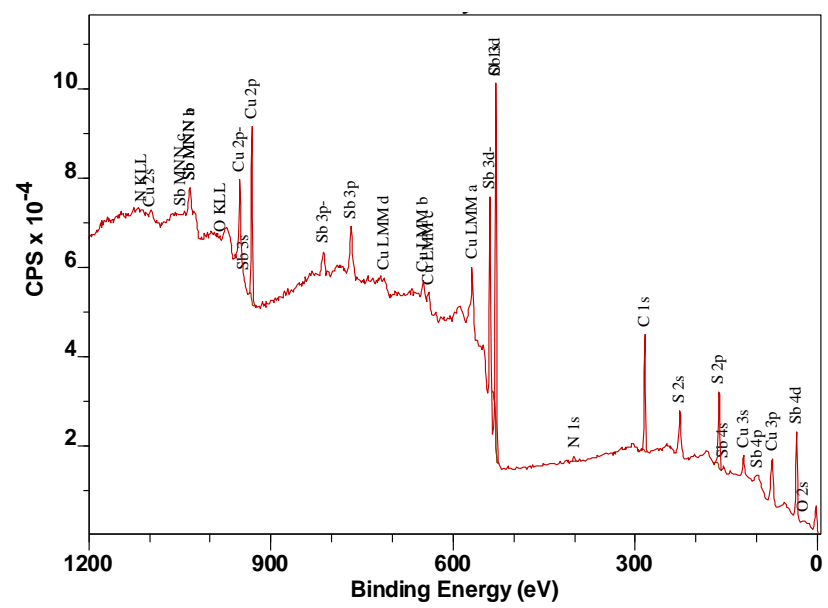

Fig. 6. XPS spectrum of CAS nanoparticles synthetized with excess sulfur

In the XPS spectrum, the peaks corresponding to nitrogen and carbon account for the presence of organic compounds at the surface of the nanocrystals. The spectrum also indicates the presence of oxygen. Sulfur S 2p region in the XPS spectrum is given in Fig. 7. The low intensity peak centered at $168.8 \mathrm{eV}$ indicates the presence of sulfates, which may account for the presence of oxygen in the sample. A quantitative analysis of the broad XPS spectrum indicates less than $1 \%$ sulfates in the analyzed area. 
Quantitative analysis of atomic composition of the nanoparticles surface gives an excess sulfur and antimony content compared to the $\mathrm{Cu}_{3} \mathrm{SbS}_{4}$ formula. The sulfur excess is partly attributed to the presence of sulfates at the nanocrystal surface. As sulfur and antimony have been added in excess prior to the synthesis, they may have been adsorbed on the surface of the nanoparticles.

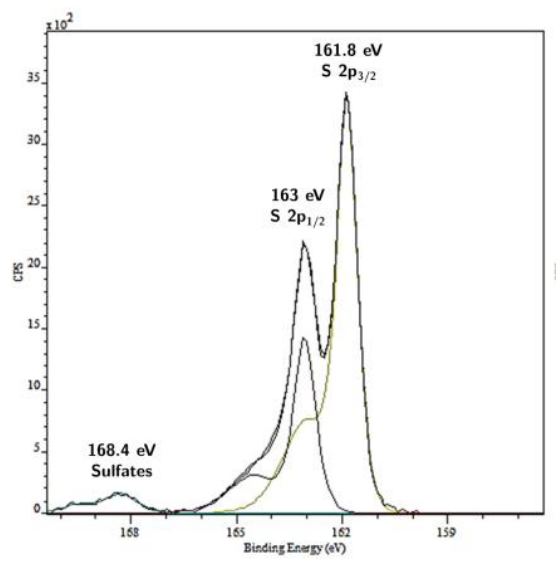

Fig. 7. XPS S $2 p$ spectrum of famatinite NCs

Ink preparation from functionalized NCs. After the purification process nanocrystals are found to be readily dispersible in non-polar solvents such as n-heptane and hexane. XPS spectrum of phase pure famatinite nanocrystals in which the peak at $399 \mathrm{eV}$ can be assigned to the amine group $\left[\mathrm{R}-\mathrm{NH}_{2}\right]$ (Fig. 8.) confirms the presence of oleylamine. The peak centered at $401.7 \mathrm{eV}$ can be attributed to oxidized $\mathrm{N}$ groups.

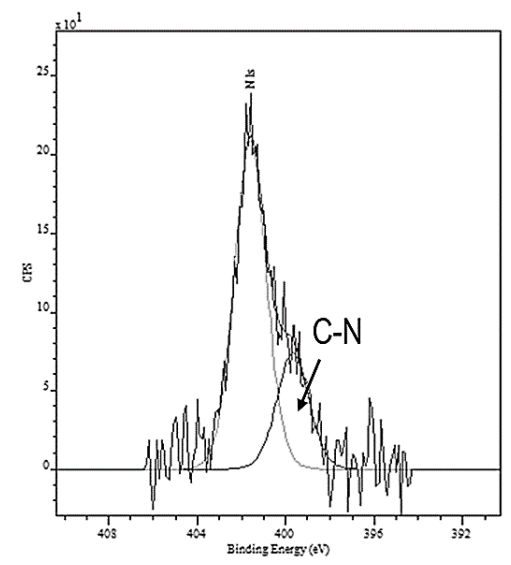

Fig. 8. XPS N 1s core level spectrum from famatinite NCs analysis

No precipitate is observed in the $50 \mathrm{wt} \%$ dispersion after a few hours of storage. After a few days of shelf storage, sedimentation of nanoparticles can be observed. The homogeneity can be restored after a few minutes of ultra-sonication. One can explain this phenomenon by the fact that the bonds between the inorganic nanoparticle surface and an electron-donating end group of a ligand molecule, such as thiol ${ }^{14-15}$, amine or phosphine ${ }^{16}$, undergo dynamic binding and unbinding processes ${ }^{17-18}$. This yields to the important consequence that the ligand molecules can get off for instance by excessive washing, which might compromise the stability of the nanoparticles that might ultimately aggregate and precipitate over time.

To overcome this issue, we added a dispersing agent, Solsperse ${ }^{\circledR} 9000$ from Lubrizol, up to 1 wt\% which was found to be benefic for the obtention of an homogeneous and stable dispersion (Fig. 9.). 


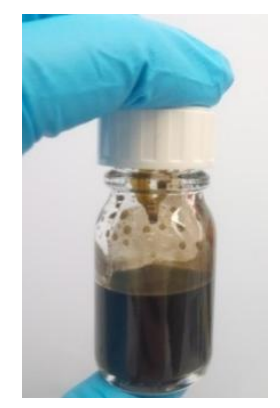

Fig. 9. Famatinite NCs based dispersion

After a few weeks of storage, sedimentation is observed, the dispersion has thus to be sonicated before use. Increasing the amount of the dispersing agent induces better stability over time. However, the addition of more than $2 \mathrm{wt} \%$ of dispersing agent leads to highly resistive films after a deposition and drying step.

The prepared solution is found to be easily processable and is therefore used for the fabrication of CAS films for further characterization.

CAS films morphological study. According to the methodology described in the experimental section, CAS films have been deposited on quartz substrates. Homogeneously deposited films are obtained via dynamic spin coating. Fig. 10.-a shows a top view of the as deposited film and Fig. 10.-b shows a cross section of the same film. To obtain the cross section, the sample is etched via low ion polishing using an Ilion Gatan system.

SEM images in Fig. 10 show a top and cross-section view of a spin-coated film on a glass substrate. The spin-coated film is found to be crack free and homogeneous with a thickness of $2 \mu \mathrm{m}$. We can observe an inhomogeneous layer formed on top of the CAS film.

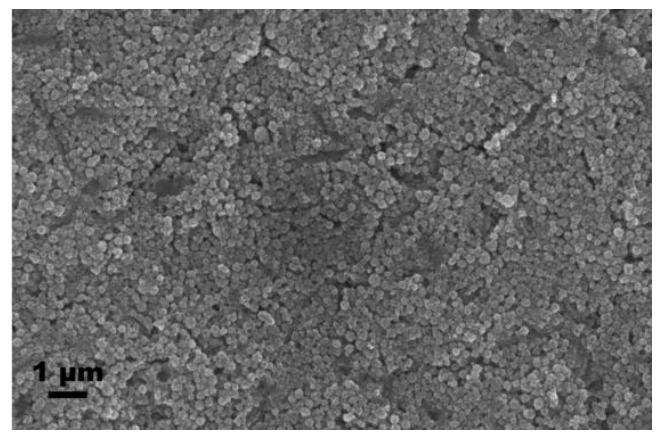

(a)

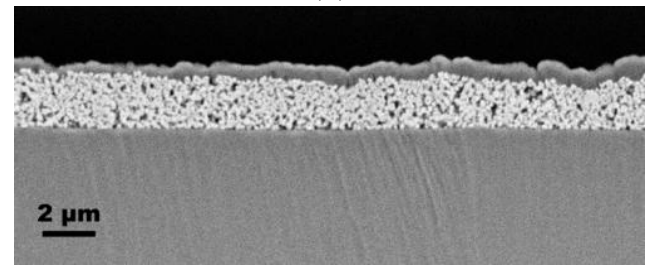

(b)

Fig. 10. SEM images of (a) top and (b) crosssection views of the spin-coated layer

From EDS measurement, this layer corresponds to the deposition of glass, from the substrate, during the etching process. The spin coated film thickness is homogeneous.

CAS films electrical and optical characterization. Spin coated films are found to be conductive, albeit highly resistive with a sheet resistance around $240 \mathrm{k} \Omega / \square(\rho=48 \Omega . \mathrm{cm})$. Lower resistivities are achieved for drop deposited films, around $10 \Omega . \mathrm{cm}$, despite the presence of micro 
cracks. The measured resistivity is higher than reported value of $0.249 \Omega . \mathrm{cm}$ for bulk famatinite ${ }^{19}$. The relatively high resistivity of the nanocrystals based films can be due to different factors such as nanoparticles cristallinity, high density grain boundaries and presence of organic compounds at some interfaces between nanocrystals (surfactant and dispersing agent). As our nanoparticles show good crystallinity similar to that of K. Ramasamy et al. ${ }^{9}$, the high resistivity is likely attributable to the two latter factors. Thicker layers deposition along with a sintering step may be useful to further reduce the electrical resistivity of the famatinite film.

Optical characteristics of spin coated CAS films on glass substrates are then characterized through UV/VIS/NIR spectroscopy in the spectral range from $200 \mathrm{~nm}$ to $1400 \mathrm{~nm}$. Optimal absorbance without saturation is obtained for $100 \mathrm{~nm}$ thick films (see Fig. 11).

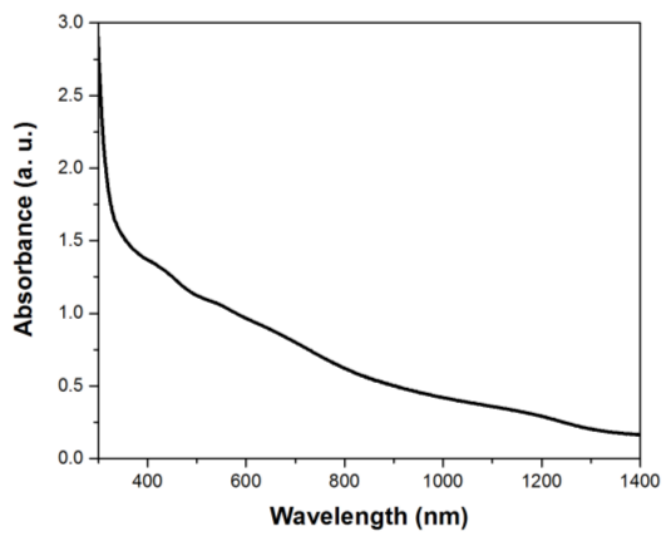

Fig. 11. UV/VIS/NIR absorption spectrum of famatinite NCs based $100 \mathrm{~nm}$ thick film

The optical band gap of films can be calculated using the Tauc relation ${ }^{20}$, that reads:

$$
(\alpha h v)^{n}=C \cdot\left(h v-E_{g}\right)
$$

Where $C$ is a constant of proportionality, $h$ is Planck's constant, $v$ is the frequency of vibration, $\alpha$ is the absorption coefficient and $n$ depends on band transitions. For a direct transition, $\mathrm{n}$ equals 2 and for an indirect one, $\mathrm{n}$ equals $1 / 2$. Based on the UV/VIS spectrum, both $(\alpha h v)^{2}$ and $(\alpha h v)^{0.5}$ are plotted as a function of $h v$. Then, a fit of the linear portion of the curve and an extrapolation at $\alpha=0$ is performed. For famatinite (batch-1.1), the extrapolated energy gap is found to be $0.82 \mathrm{eV}$ with an indirect transition (Fig. 12). The plot of $(\alpha h v)^{2}$ versus $h v$ gives a higher energy gap of $1.5 \mathrm{eV}$ for the direct transition.

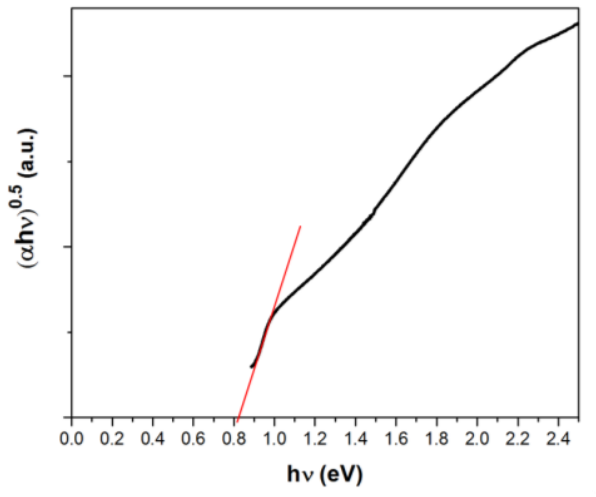

(a)

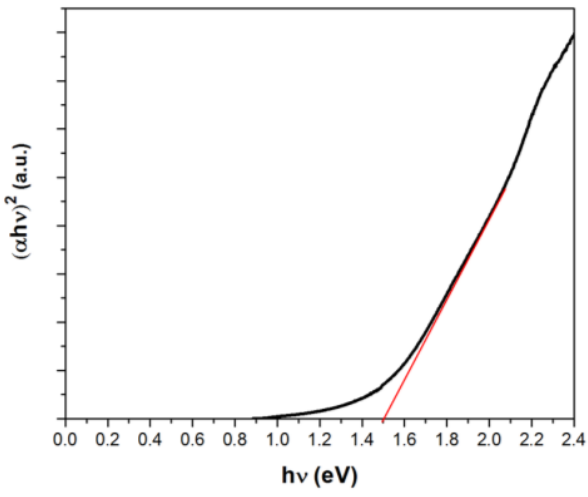

(b) 
Fig. 12. Dependence of (a) $(\alpha h v)^{0.5}$ and (b) $(\alpha h v)^{2}$ on photon energy $h v$ plot for famatinite NCs based $100 \mathrm{~nm}$ thick film

The calculated band gap is in the range of reported values for famatinite, from $0.46 \mathrm{eV}$ to $1.2 \mathrm{eV}^{6,9,19}$, 21, 22. The literature data is however ambiguous as both direct ${ }^{19,21}$ and indirect band gap ${ }^{9,22}$ are reported for $\mathrm{Cu}_{3} \mathrm{SbS}_{4}$.

\section{Conclusion}

Famatinite nanocrystals were synthetized by a surfactant assisted solvothermal method under mild and safe conditions as no toxic solvents and precursors were used. Nanocrystalline and phase-pure famatinite particles were obtained by carefully controlling the surfactant to solvent volumetric ratio as well as the sulfur to copper molar ratio and without the need of an inert atmosphere during the synthesis process. The structure of the synthetized $\mathrm{Cu}_{3} \mathrm{SbS}_{4}$ nanocrystals was confirmed by XRD. For the synthesis, excess of sulfur in the molar ratio $\mathrm{S}: \mathrm{Cu}$ was necessary to obtain the phase-pure famatinite, whereas the stoichiometric ratio 1:0.75 yielded a famatinite-tetraedrite mix of phases. The proposed method is easily scalable as compared to the hot-injection method partly because all the precursors are mixed before the reaction. A homogeneous and easily processable solution was obtained. Depending on the printing technique used to deposit the nanoparticle based solution, further formulation optimization can be done to adjust key parameters such as viscosity and nanoparticle size. The prepared dispersion was used to fabricate homogeneous films. The electrical resistivity of the films could be reduced by increasing their thickness. Further studies on the impact of sintering on the electrical properties have to be conducted to evaluate the possibility to improve the electrical conductivity of famatinite films.

\section{Acknowledgements}

This work has been supported by an ANRT CIFRE grant. The authors would like to thank L. Beauvisage and L. Fares from STMicroelectronics Rousset (RCCAL laboratory) for their support on TEM characterizations as well as B. Bortolotti from STMicroelectronics Rousset (RCCAL laboratory) for the XPS analysis. The authors would also like to thank C. Chassigneux from MADIREL laboratory for the help with XRD analysis.

\section{References}

(1) Gur, I.; Fromer, N. A.; Geier, M. L.; Alivisatos, A. P. Science, 2005, 310, 462.

(2) Kamat, P. V. J. Phys. Chem. C, 2008, 112, 18737.

(3) Jasieniak, J.; MacDonald, B. I.; Watkins, S. E.; Mulvaney, P. Nano Lett., 2011, 11, 2856.

(4) Poudel, B.; Hao, Q.; Ma, Y.; Lan, Y.; Minnich, A.; Yu, B.; Yan, Z.; Wang, D.; Muto, A.; Vashaee, D.; Chen, X.; Liu, J.; Dresselhaus, M. S.; Chen, G.; Ren, Z. Science, 2008, 320, 634.

(5) Wang, R. Y.; Feser, J. P.; Jong-Soo, L.; Talapin, D. V.; Segalman, R.; Majumdar, A. Nano Lett. $2008,8,2283$.

(6) J. van Embden, Y. Tachibana, J. Mater. Chem., 2012, 22, 11466

(7) J. van Embden, K. Latham, N. W. Duffy, Y. Tachibana, J. Amer. Chem. Soc., 2013, 135, 11562

(8) D. Xu, S. Shen, Y. Zhang, H. Gu, and Q. Wang, Inorg. Chem., 2013, 52 (22), 12958-12962 
(9) K. Ramasamy, H. Sims, W. H. Butler, and A. Gupta, Chem. Mater., 2014, 26 (9), 2891-2899.

(10) C. An, Y. Jin, K. Tang and Y. Qian, J. Mater. Chem., 2003, 13, 301-303

(11) J.W. Thomson, K. Nagashima, P.MacDonald and G. A. Ozin, J. Am. Chem. Soc., 2011, 133 (13), 5036-5041

(12) S. Mourdikoudis and L. M. Liz-Marzań, Chem. Mater., 2013, 25, 1465-1476

(13) Wang, S., An, C., Yuan, J., Materials, 2010, 3, 401-433

(14) C. S. Weisbecker, M. V. Merritt, G. M. Whitesides, Langmuir, 1996, 12 (16), 3763-3772

(15) S. Lin, Y. Tsai, C. Chen, C. Lin, C. Chen, The Journal of Physical Chemistry B, 2004, 108 (7), 2134-2139

(16) D. V. Leff, L. Brandt, J. R. Heath, Langmuir, 1996, 12 (20), 4723-4730

(17) H. Döllefeld, K. Hoppe, J. Kolny, K. Schilling, H. Weller, A. Eychmüller, Phys. Chem. Chem. Phys., 2002,4, 4747-4753

(18) X. Ji, D. Copenhaver, C. Sichmeller, X. Peng, Journal of the American Chemical Society, 2008, 130 (17), 5726-5735

(19) Y. Goto, Y. Sakai, Y. Kamihara, and M. Matoba, J. Phys. Soc. Jpn., 84, 044706 (2015).

(20) J. Tauc and A Menth, J. Non-Crystalline Solids, 1972, 8-10

(21) C. T. Crespo, J. of Phys. Chem. C, 2016, 120 (15), 7959-7965

(22) S. Suehiro, K. Horita, M. Yuasa, T. Tanaka, K. Fujita, Y. Ishiwata, K. Shimanoe, and T. Kida, Inorg. Chem., 2015, 54 (16), 7840-7845 